PREPRINT:

\title{
Areal parameter estimates from multiple datasets
}

\author{
B.L.N. Kennett \\ Research School of Earth Sciences, The Australian National University, Canberra, Australia \\ email: brian.kennett@anu.edu.au
}

Keypoints

Combination of multiple datasets to delineate spatial variation

Points in a single dataset assigned a spatial influence zone and relative weighting

Weighting between datasets to reflect data reliability.

\begin{abstract}
A wide range of methods exist for interpolation between spatially distributed points drawn from a single population. Yet often multiple datasets are available with differing distribution, character and reliability. A simple scheme is introduced to allow the fusion of multiple datasets. Each dataset is assigned an a priori spatial influence zone around each point and a relative weight based on its physical character. The composite result at a specific location is a weighted combination of the spatial terms for all the available data points that make a significant contribution. It is therefore also useful for sparse observations that characterise a limited spatial zone, such as heat flow. The combination of multiple data sets is illustrated with the construction of a unified Moho surface in part of southern Australia from results exploiting a variety of different styles of analysis.
\end{abstract}

\section{INTRODUCTION}

Many procedures for constructing surface representations of geophysical information are based on the assumption that the available samples are drawn from a single stochastic population. Such concepts underlie kriging methods (e.g. Oliver, 1990) and their extension to gaussian processes (e.g. Williams et al., 1998). Other spatial interpolation schemes are designed to generate a surface that passes directly through a sparse set of sample points, with assumptions about the fitting surface such as minimum curvature (e.g. Smith \& Wessel, 1990). A useful summary of many different methods of spatial interpolation and their limitations is provided by Mitas \& Mitasova (1999). 
However, in some circumstances there can be multiple spatial estimates for a parameter with different distributions and reliabilities, e.g., from surveys carried out at various times with different equipment. It is possible to compensate to some extent by introducing relative weighting between data points in a single mode of interpolation.

The situation becomes even more complicated when data estimates are based on different physical approaches, as occurs e.g. in the representation of the depth to Moho. A number of methods provide information on the crust-mantle boundary such as the interpretation of refraction and reflection surveys, receiver functions and autocorrelation techniques. Multiple methods may also be used at the same seismic stations.

The approach used by Kennett et al., (2011) to construct a Moho surface across the Australian continent was to assign specific weights to each observation, and combine all results in a $0.5^{\circ} \times 0.5^{\circ}$ pixel with the weighted average assigned to centre of each pixel. These new values were then used as the basis for interpolation using an adjustable tension continuous curvature gridding algorithm (Smith \& Wessel, 1990). This data averaging suppresses the local information present in, e.g., full-crustal reflection profiling. Further when the only result in a pixel is assigned low weight, this value will be transmitted directly into the continent wide surface.

Since 2011 the number of Moho estimates in Australia has grown significantly, with a strong continuing program of full-crustal reflection profiling and the advent of new methods such as the exploitation of stacked station autocorrelograms (Kennett et al, 2015; Qashqai et al, 2019). In many places this means that there are multiple values for the depth to Moho based on different physical assumptions.

We here propose a new scheme in which we take account of the nature of different classes of observation, not only with data weights but with varying spatial influence zones. The relative weighting of different styles of datasets can also be adjusted. The resulting expression for the data estimate at any spatial point has a formal similarity to kriging and other single dataset interpolators, but combines results from multiple datasets.

\section{EXPLOITING MULTIPLE DATASETS}

With a suite of data sets $\{d\}$, we assign internal weights $w_{k d}$ to each data point $m_{k d}$ in dataset $d$. All points in the same data set are then assigned a spatial influence depending on the nature and reliability of the data. We here use radial Gaussian spread functions with a scale length $\sigma_{d}$ to describe the spatial patterns, building on the work of Kennett et al. (2015) who used such weighting for spatial stacking of data from many seismic stations in southeastern Australia. 
We combine the information from different data sets allowing for relative weighting between data sets specified by a weight $w_{d}$ that is data set specific. We then build the estimate of the local value of the field at the $i$ th spatial point $M_{i}$ from a sum of all contributions with allowance for weighting and the distance of the data points from the sample point:

$$
M_{i}=\frac{\sum_{d} w_{d} \sum_{k} w_{k d} m_{k d} e^{-\left(\Delta_{i k} / \sigma_{d}\right)^{2}}}{\sum_{d} w_{d} \sum_{k} w_{k d} e^{-\left(\Delta_{i k} / \sigma_{d}\right)^{2}}},
$$

where $\Delta_{i k}$ is the distance from the $i$ th sample point to the observation. In principle, all data points contribute to an estimated value, but it is expedient to truncate each spatial spread at a spatial separation of $3.5 \sigma_{d}$ to avoid cumulation of numerical truncation error from exceedingly small weights.

The resulting formula for the spatial estimate has comparable structure to interpolation with inverse distance weighting (Watson \& Philip, 1985) or kriging (Oliver, 1990), but the weights are determined a priori based on the nature of each measurement rather than being determined by the data point distribution. The representation (1) also allows for multiple data classes with their own character, rather than assuming all points drawn from a single random variable distribution as in kriging.

The concept of the weighted spatial-spread procedure is illustrated in Figure 1, where we show the construction of a single surface from a combination of three datasets. The first dataset comprises a limited number of data points with high reliability that have broad spatial sampling; the second data set has medium quality and spread with greater variation in reliability and the third rather dense data of variable quality and narrow spatial influence. In the upper three rows of Figure 1 we plot the spatial spread assigned to each data point colour coded by the actual data value. The bottom row shows the resulting distribution when all the data points are combined using the weighted spatial-spread formula (1) at a dense line of sample points. In this simple example each distinct dataset is assigned equal weight, and only the internal weights are taken into consideration.

The weighted spatial-spread result (1) is simple to implement and is very versatile. The estimator may be made with absolute data values or in relative mode where one examines deviations from a reference value or a predefined reference surface. When used with just a single dataset it can provide a smoothing interpolator with the degree of smoothness controlled by the assigned spatial spread. Alternatively, one can represent spatially distinct points with combination of results only where the influence zones overlap. Such an approach works well for sparse observations such as heat flow that characterise a limited spatial zone.

\section{APPLICATION: MULTIPLE MOHO DATASETS}

As an example of the application of the weighted spatial-spread approach we present results for estimates of the depth to Moho for an area of southern Australia. This region has a broad range of different 

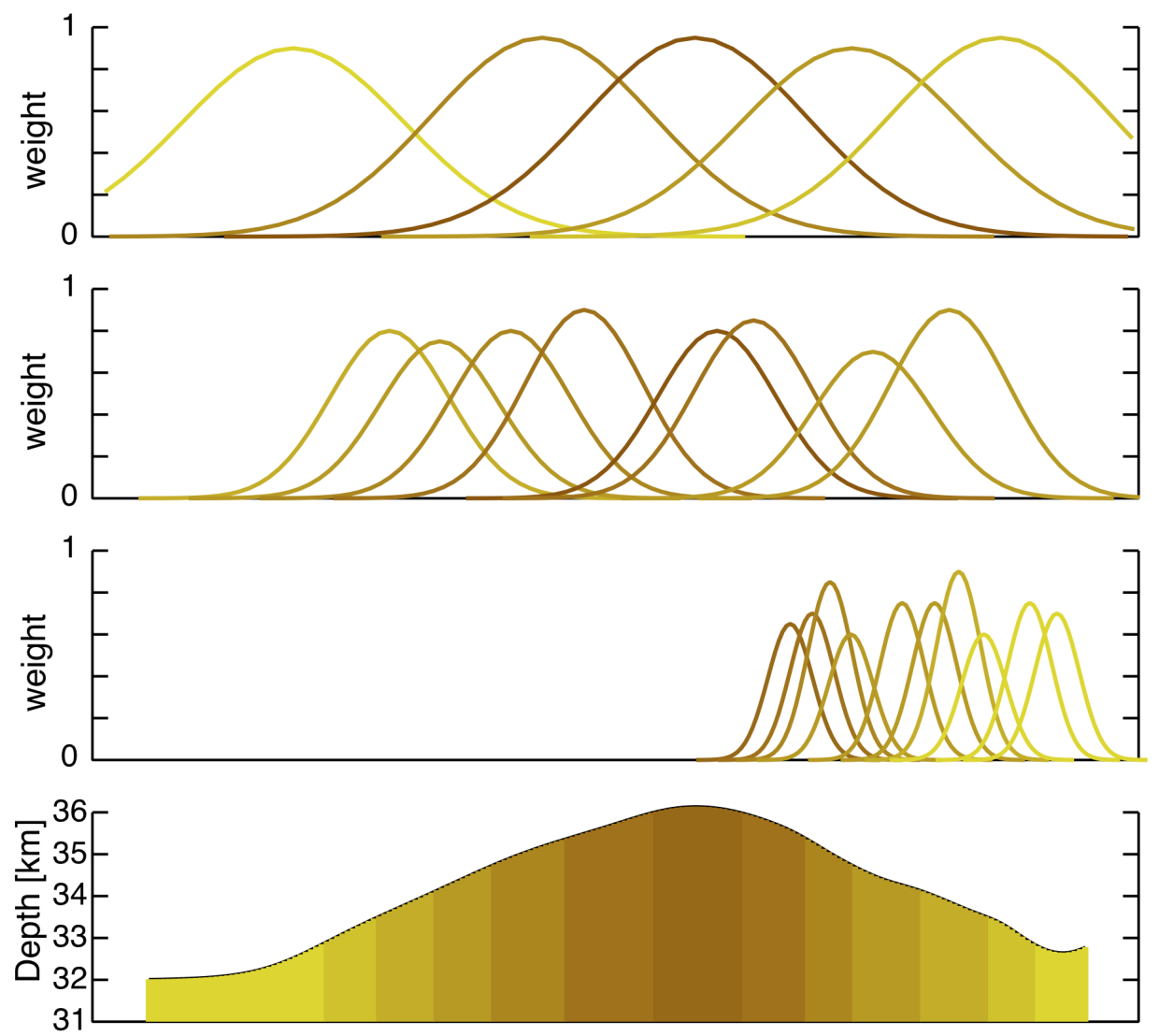

Figure 1. Application of weighted spatial spread method to three distinct data sets with variable spatial influence and reliability, as indicated on the top three rows, to produce a single spatial surface shown on the bottom line. The data points are colour coded by their value. The spatial extent and weight for each is drawn directly.

data samples with an uneven data distribution. In Figure 2 we show the full range of data points, color coded by Moho depth with symbols that indicate the nature of the measurement scaled to indicate the reliability of the results and thus the weights applied.

In Table 1 we show the relative weight assigned to the datasets and the spatial spreads used in creating Figures 3 and 4 . The spread estimates are based on the spatial sampling associated with each data class. Thus refraction experiments average over more than $100 \mathrm{~km}$, whereas receiver based techniques are more localised. Results from full-crustal reflection surveys are available at much closer spacing, but show more variation in data quality and involve uncertainty in conversion between reflection time and depth. The spatial spread assigned to reflection data is designed to preserve such features as abrupt changes in crustal thickness (cf. Kennett \& Saygin, 2015).

In addition to these point estimates of crustal thickness, we have made use of the gravity inversion of Aitken et al. (2013) using samples on a $0.5^{\circ} \times 0.5^{\circ}$ grid. These inversion results are assigned a uniformly low weight (0.4). Figure 3 shows the results for each of the major classes of data with 


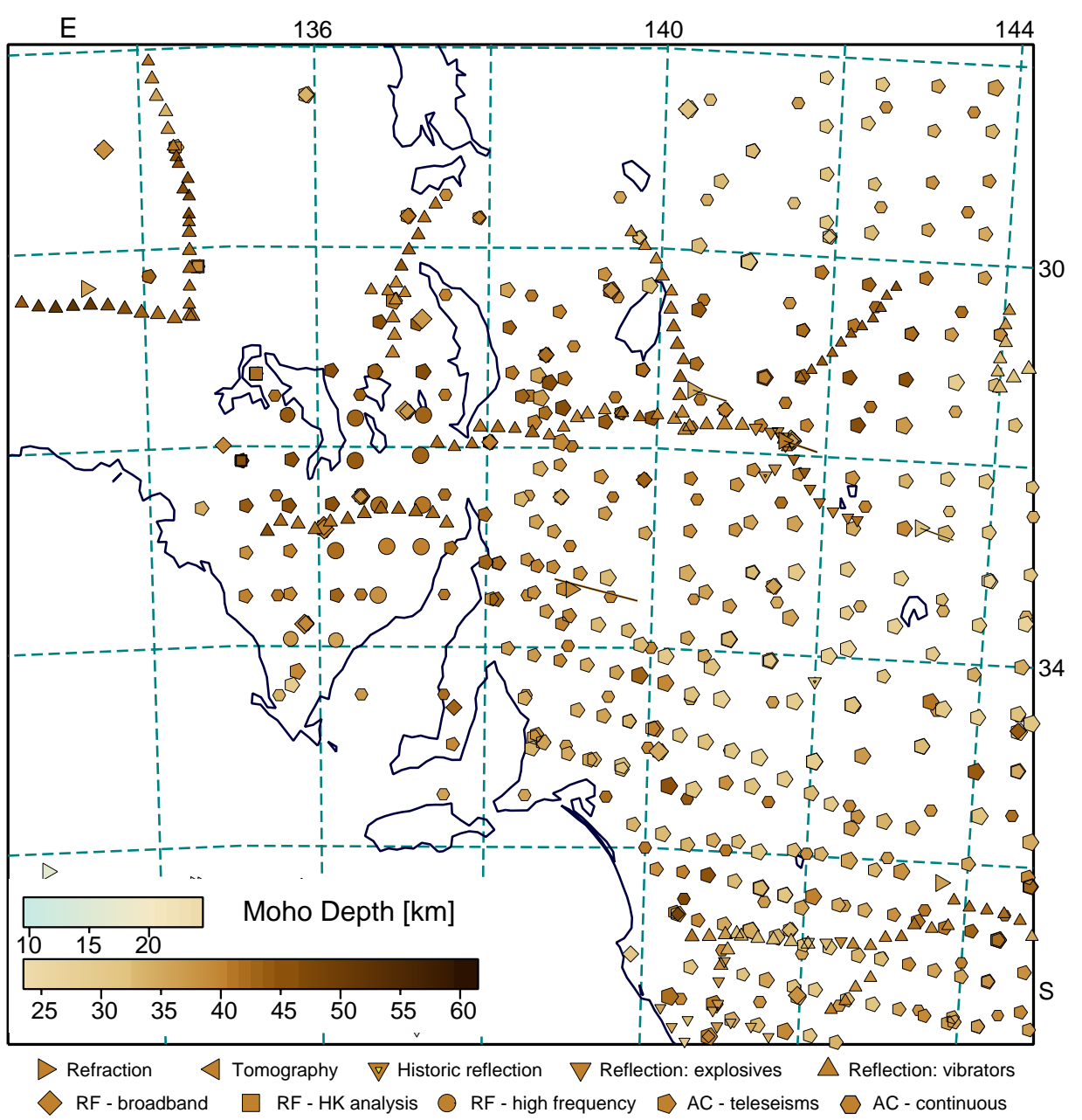

Figure 2. Distribution of Moho estimates across part of southern Australia. The points are colour-coded by depth with symbol shape determined by measurement type, scaled by reliability.

Table 1. Weighting used for multiple datasets

\begin{tabular}{lcc} 
Data type & weight & spatial spread $\left[{ }^{\circ}\right]$ \\
\hline refraction & 1.0 & 1.2 \\
marine wide-angle & 1.0 & 0.4 \\
receiver functions & 1.0 & 0.6 \\
tomography & 1.0 & 0.6 \\
autocorrelation & 0.9 & 0.5 \\
reflection & 0.9 & 0.2 \\
gravity inversion & 0.5 & 0.4
\end{tabular}




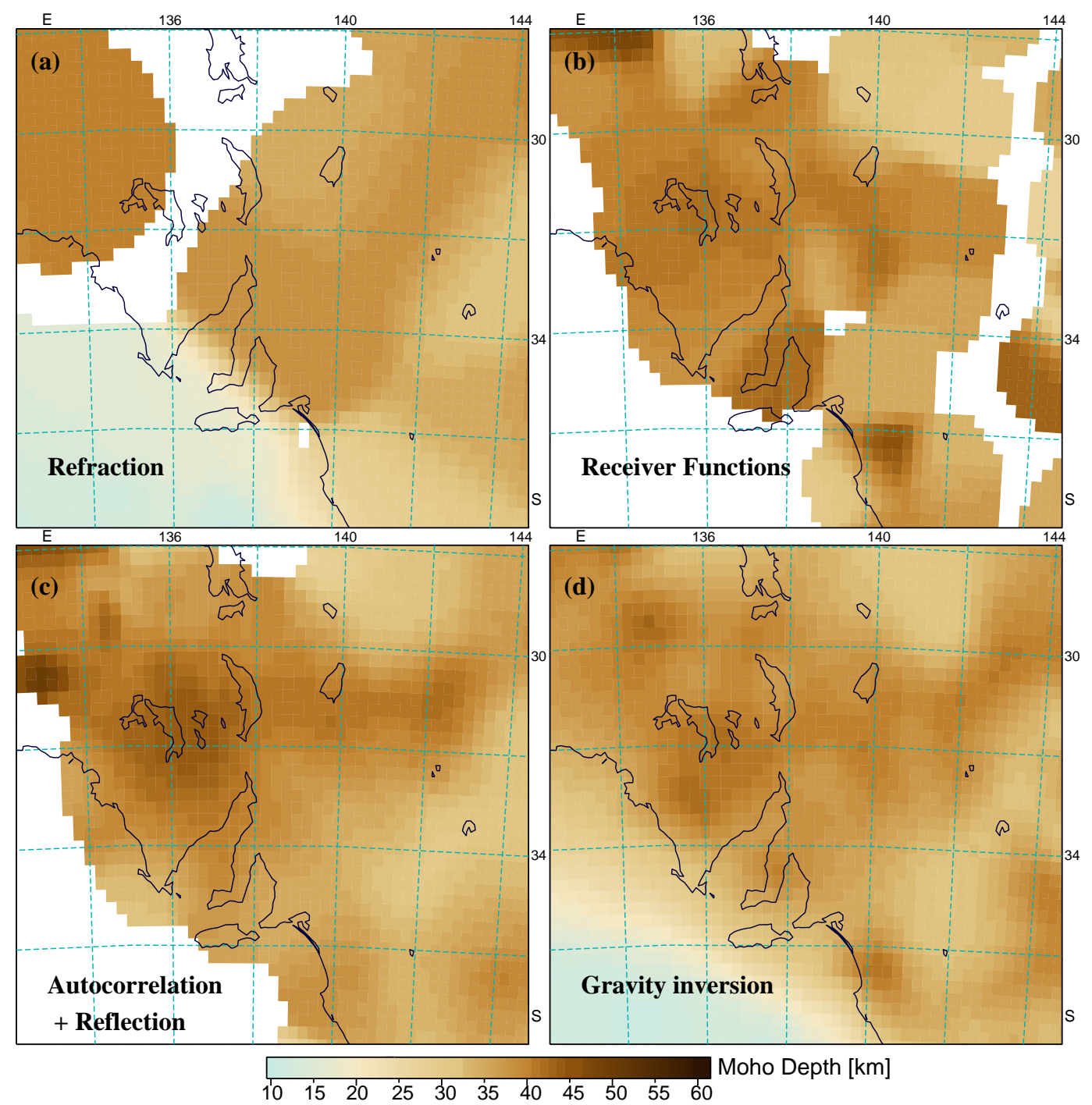

Figure 3. Estimates of Moho depth using the weighted spatial spread approach for restricted data sets: (a) refraction and marine wide-angle information; (b) receiver functions; (c) autocorrelation and reflection profiling and (d) gravity inversion.

truncation of the estimates at the same weighting threshold (0.02). None of the point data achieves full coverage of the region. Even when all this data is combined there are some patches where no direct estimation can be made, and considerable areas where low weight results have to be employed (Figure $4 a)$.

If we add in the results from the gravity inversion with very low weight (combined weighting 0.2), we achieve a better rendering for the whole domain (Figure $4 \mathrm{~b}$ ). The presence of the results from the gravity inversion has a large impact on the resolution of the continent-ocean transition, but also gives an improved result in areas with limited data coverage since it fills in the holes.

In Figure 5 we display the combination of all the different datasets and superimpose the original 


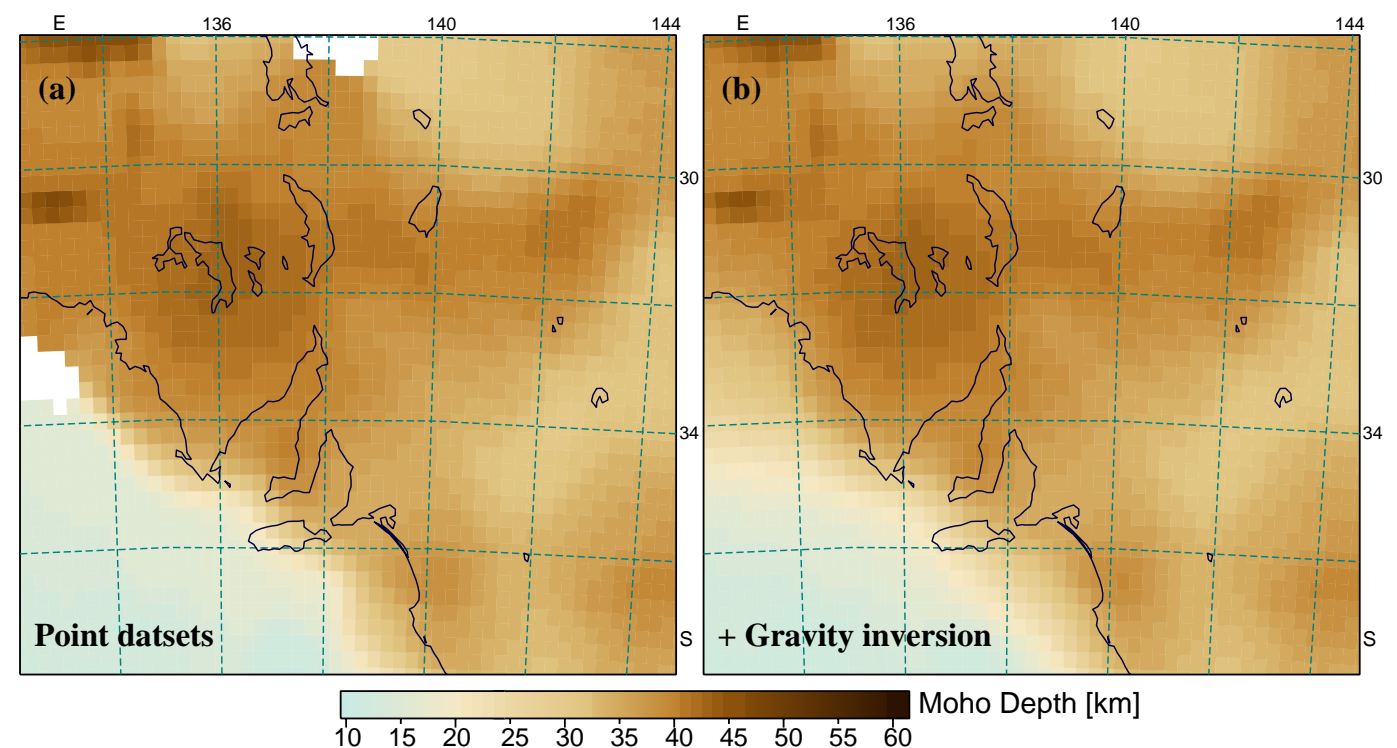

Figure 4. Estimates of Moho depth using the weighted spatial spread approach: (a) all 9 sets of point data and (b) with the inclusion of a low weight applied to gravity inversion.

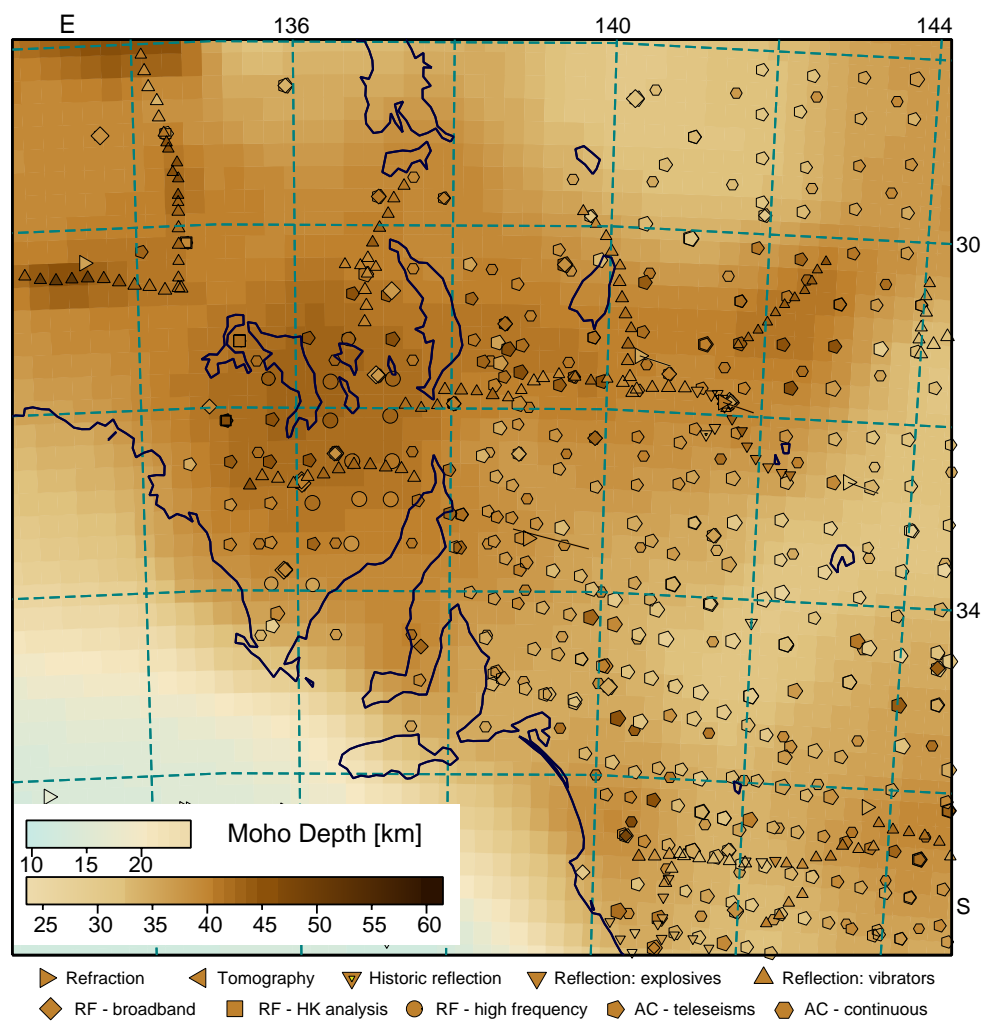

Figure 5. Comparison of the Moho surface derived by combining all the various classes of information using the weigh spatial spread approach with the original data values. 
data values from Figure 2. We note that a number of the autocorrelation estimates using earthquake signals remain discordant, even though they have influenced the construction of the Moho surface. The physical model used to extract these results enforces a sharp discontinuity (Qashqai et al., 2019), and the differences from the interpreted surface, where present, provide an indication of the thickness of the crust-mantle transition.

\section{DISCUSSION AND CONCLUSIONS}

We have established a procedure that enables multiple data sets to be combined to produce a single spatial surface. The approach is simple to implement and produces a very flexible scheme, since the character of the combination is under full user control through a priori choices for the weighting of data points and the spatial spread around each point assigned to each data set. This means that different choices for the relative weighting of different datasets can readily be explored. Indeed it is possible to look at the uncertainties associated with the inclusion of particular datasets by setting up an ensemble of surface constructions.

It is desirable that the spatial influence zone assigned to each class of observation conforms to the physical nature of the actual measurement, rather than be arbitrarily extended. The use of such confined influence zones may mean that parameter estimates are more spatially restricted than in a standard broad-scale interpolation, but they will represent the direct impacts of the measurements. In this context it can be useful to employ a background field that can be assigned low weight to compensate for data holes, as in the use of the gravity inversion results in the Moho results in the example.

In the treatment above we have used radial Gaussian spread functions around each data point, but these functions could be replaced by other spatial samplers if they are more appropriate to different classes of information. For data that has a strongly directional character it may well be worthwhile to use a spatial influence zone with an elliptical footprint defined by two scale lengths and a preferred axis of orientation. If slower spatial decay is desired then a simple exponential of spatial separation can replace the quadratic form.

\section{ACKNOWLEDGMENTS}

The assembly of the many different Moho datsets has involved a wide range of contributors over the years and all are thanked for their efforts. The Moho data values are lodged at the Australian seismological data repository (auspass.edu.au). 


\section{REFERENCES}

Aitken, A.R.A., M.L. Salmon and B.L.N. Kennett, (2013) Australia’s Moho: A test of the usefulness of gravity modelling for the determination of Moho depth, Tectonophysics 609, 468-479.

Kennett, B.L.N., M. Salmon, E. Saygin and AusMoho Working Group, (2011) AusMoho: the variation in Moho depth in Australia, Geophys. J. Int. 187, 946-958.

Kennett, B.L.N. and E. Saygin, (2015) The nature of the Moho in Australia from reflection profiling: A review, GeoResJ 5, 74-91.

Kennett, B.L.N., E. Saygin and M. Salmon, (2015) Stacking autocorrelograms to map Moho depth with high spatial resolution in southeastern Australia, Geophys. Res. Lett. 42, 3839-3846.

Mitas, L. and H. Mitasova (1999) Spatial interpolation, inGeographical Information Systems: Principles, Techniques, Management and Applications (2nd edition), Eds: P.A. Longley, M.F. Goodchild, D.J. Maguire and D.W. Rhind, Wiley.

Oliver, M. A. (1990) Kriging: A Method of Interpolation for Geographical Information Systems. Inter. J. Geographic Information Systems 4, 313-332.

Qashqai, M.T., E. Saygin and B.L.N. Kennett, (2019) Crustal imaging with Bayesian inversion of P-wave coda autocorrelation, J. Geophys. Res.

Smith, W.H.F. and P. Wessel, (1990) Gridding with continuous curvature splines in tension, Geophysics 55, 293-305.

Watson, D. F., and G. M. Philip. (1985) A Refinement of Inverse Distance Weighted Interpolation. Geoprocessing 2, 315-327.

Williams, C. K. I. (1998). Prediction with Gaussian Processes: From Linear Regression to Linear Prediction and Beyond. Learning in Graphical Models. Ed. M.I. Jordan, pp. 599-621, Kluwer, Dordrecht. doi:10.1007/978-94-011-5014-9_23 